\title{
Strates
}

STRATES Matériaux pour la recherche en sciences sociales

$7 \mid 1993$

Témoins du monde : Bulgarie, identités chinoises, explorer l'île de France

\section{Les nouvelles communes au Brésil : trois expériences d'émancipation dans une zone de modernisation agricole}

\section{Zila Mesquita}

\section{(2) OpenEdition}

\section{Journals}

Édition électronique

URL : http://journals.openedition.org/strates/1163

DOI : $10.4000 /$ strates. 1163

ISSN : $1777-5442$

Éditeur

Laboratoire Ladyss

Édition imprimée

Date de publication : 30 juin 1993

ISSN : 0768-8067

\section{Référence électronique}

Zila Mesquita, «Les nouvelles communes au Brésil : trois expériences d'émancipation dans une zone de modernisation agricole », Strates [En ligne], 7 | 1993, mis en ligne le 20 décembre 2005, consulté le 08 septembre 2020. URL : http://journals.openedition.org/strates/1163 ; DOI : https://doi.org/

$10.4000 /$ strates. 1163

Ce document a été généré automatiquement le 8 septembre 2020

Tous droits réservés 


\title{
Les nouvelles communes ${ }^{1}$ au Brésil : trois expériences d'émancipation dans une zone de modernisation agricole
}

\author{
Zila Mesquita
}

\section{NOTE DE L'ÉDITEUR}

Traduit du brésilien par Michel Rochefort et Michèle Petit. L'auteur remercie Clelia Lustosa et Jose Borzacchiello da Silva, géographes et professeurs à l'Université fédérale du Ceara, pour leurs critiques et suggestions.

1 Actuellement, $70 \%$ de la population brésilienne vit déjà dans des villes. Ce processus d'urbanisation est croissant et continu, comme le montrent les recensements démographiques des quatre dernières décennies. De surcroît, ce processus est rapide : en 1960, la part de la population urbaine dans la population totale était de $44,9 \%$ et elle est passée vingt ans plus tard à $67,6 \%$.

Parallèlement, la dernière décennie a vu l'émergence de divers mouvements luttant en faveur d'une "émancipation " par la création de nouvelles communes, ou même de nouveaux États au sein de la Fédération brésilienne. Les habitants des zones urbaines et surtout suburbaines savent combien les carences en services, en logements et en approvisionnement collectif rendent leur vie quotidienne difficile. Mais de telles carences touchent aussi les agriculteurs des environs qui ont également besoin de services que les villes, en principe, devraient pouvoir leur fournir. Dans ce sens, l'espace urbain brésilien exprime aujourd'hui tous les drames de la société, et c'est ainsi un espace-synthèse ${ }^{2}$.

La « modernisation douloureuse » 
3 Les analyses relatives à l'évolution récente de la réalité brésilienne se rejoignent sur plusieurs points. Elles reconnaissent que l'économie brésilienne a connu après-guerre un processus de modernisation, tant dans l'industrie que dans l'agriculture. Dans le cas de l'agriculture, ce processus, selon certains, aurait pris la forme d'une « modernisation conservatrice $»^{3}$. Ces auteurs affirment qu'elle est conservatrice sur le plan social, car la pénétration de l'agriculture par le capital n'altère pas la structure foncière par le biais d'une meilleure distribution des terres. Au contraire, elle maintient la concentration de la propriété entre les mains d'un petit nombre, conformément à l'ancien modèle oligarchique, et elle exclut et spolie la majorité des petits agriculteurs. D'autres réfutent cet aspect conservateur, parce que cette modernisation aboutit à une injection de capital dans l'agriculture. Certains, comme Graziano da Silva, préfèrent parler de "modernisation douloureuse", du fait de son caractère excluant ${ }^{4}$. Mais le point essentiel, c'est que la rapidité de l'urbanisation dans le pays est due à l'importance accordée à la modernisation des activités économiques.

4 A partir des années cinquante, la région sud, et particulièrement l'intérieur de l'État de Sao Paulo et le nord du Rio Grande do Sul, dans le cas de l'agriculture, ont servi d'«espaces-tests» pour cette modernisation, par le développement des cultures capitalistes de blé et de soja ${ }^{5}$. La modernisation a ensuite atteint le Parana, avant de s'étendre, dans les années soixante-dix, à la région du Centre-Ouest et à l'Amazonie et, plus récemment, à l'intérieur de l'État de Bahia. C'est dans l'un de ces « espaces-tests », le nord du Rio Grande do Sul, que se situent les trois nouvelles villes étudiées dans le cadre de cette recherche.

5 Il faut signaler que cet espace a été une zone d'expulsion de population depuis plusieurs décennies, les migrants se dirigeant vers l'aire métropolitaine de Porto Alegre, ou vers les régions du pays mentionnées plus haut. Cette migration, si elle est peu souhaitable dans les grandes agglomérations métropolitaines, s'avère nécessaire à l'expansion du capitalisme dans les zones d'avancée de la frontière agricole du nord du pays, et à la modernisation de l'agriculture dans les zones de départ. Dans les campagnes, elle se traduit notamment par l'importance de la population dépourvue de terre, et dans les villes, par des contingents accrus de personnes sous-employées, sans emploi, sans abri. Celles-ci ne sont pas cantonnées aux zones métropolitaines, mais se rencontrent aussi à la périphérie des villes moyennes et le long des routes qui y conduisent. Et ce n'est pas par hasard que le Rio Grande do Sul, État de l'extrême sud du pays, a été le noyau du «Mouvement des sans-terre », car c'est l'une des zones où la modernisation est la plus douloureuse.

L'analyse de trois processus d'émancipation

6 Devant cet essor de l'urbanisation/modernisation, et pour une meilleure compréhension de cette réalité, nous avons étudié cinquante-cinq processus d'émancipation qui ont été examinés par la Sous-commission des Émancipations de la Commission de la Constitution et de la Justice de l'Assemblée législative du Rio Grande do Sul. Nous avons retenu les cas d'Eugenio de Castro, Entre-Ijuis et Sao Miguel das Missos, qui relevaient initialement d'un même «municipe», Santo Angelo. Géographiquement, la question était intéressante, car si l'un de ces trois districts n'avait pas réussi à s'émanciper, il y aurait eu perte de contiguïté spatiale avec le cheflieu du «municipe », ce qui est interdit par la loi. En conséquence, il se serait créé un problème pour l'émancipation des deux autres, ou bien il aurait fallu négocier le tracé des limites. 
7 Par ailleurs, on supposait, par hypothèse, que la modernisation est partie intégrante de la régionalisation de la zone. Par régionalisation, on entend ici non l'acte de diviser l'espace selon un principe d'homogénéité ou de polarité, mais un processus de formation et de transformation des régions. Et comme tout processus, il se produit au niveau de la réalité 6 . La régionalisation suppose ainsi des transformations de la structure productive régionale. Dans les cas étudiés, il s'agit de la modernisation de l'agriculture, dont les conséquences sociales ont déjà été évoquées. Mais l'une des manifestations de cette régionalisation, stimulée par la modernisation, n'est pas toujours perçue comme telle : c'est l'existence de mouvements en faveur d'une émancipation, qui ont finalement permis aux trois anciens districts de figurer parmi les 88 nouveaux « municipes » créés en 1988 dans l'État.

8 Pour mieux comprendre ces mouvements en faveur d'une émancipation, on est parti des questions suivantes :

9 - Les revendications régionales peuvent-elles être interprétées comme l'expression d'une prise de conscience et d'une volonté de participation des populations qui les ont portées ou, au contraire, reflètent-elles de nouvelles formes de mise en application et d'expansion de la production capitaliste dans les campagnes du territoire gaucho ? Ces revendications correspondent-elles à un besoin des populations qui les expriment, ou constituent-elles l'une des formes qu'emprunte le pouvoir économique pour s'imposer?

10 - Ces revendications révèlent-elles un processus d'urbanisation des campagnes, inverse ou parallèle à une urbanisation «classique » à partir des métropoles et des grandes villes?

11 A travers ces questions se structuraient plusieurs objectifs : déceler comment et pourquoi un district recherche son émancipation politico-administrative; détecter qui revendique, comment se déroule la campagne en faveur de l'émancipation, découvrir si des intérêts économiques et politiques lui sont liés; chercher à interpréter les modifications qui sont en cours dans ces espaces.

12 Le travail de terrain a été réalisé en quatre phases. Trois se sont déroulées antérieurement aux émancipations, la quatrième un an après l'installation des nouveaux chefs-lieux de «municipes ». Il a commencé par une étude exploratoire, qui a permis d'identifier les acteurs sociaux concernés. Par le biais des informations obtenues lors d'entretiens directifs ou semi-directifs, ceux-ci ont pu être regroupés en six groupes:

13 - les informateurs privilégiés, liés ou non aux émancipations (parmi eux, des techniciens ruraux, des agronomes, des professeurs, des assistants des conseillers municipaux et des commissions chargées des émancipations, un architecte de la Fondation Pro-memoria qui travaillait dans les ruines de Sao Miguel das Missoes, et avait des opinions qui différaient de celles de la Commission dont il relevait );

14 - les membres des commissions chargées des émancipations (les entretiens étant réalisés avant celles-ci);

15 - le pouvoir institué, qui comprend les maires, les vice-maires, les anciens maires, les membres des chambres municipales, les conseillers municipaux et les personnes influentes formellement mêlées au jeu politique, tant au niveau des communes initiales que des nouveaux «municipes» (entretiens effectués avant et après les émancipations) ; 
16 - les membres des directions des syndicats des travailleurs ruraux, et les associations présentes dans les syndicats (entretiens réalisés avant et après les émancipations);

17 - la population ; les habitants des sièges et des périphéries des anciens districts devenus chefs-lieux de «municipes»; les agriculteurs de l'intérieur, comprenant ceux qui habitent dans les hameaux, les écarts et en habitat dispersé ;

18 - les personnes qui pourraient s'engager dans une recherche appliquée comme des assistants techniques des syndicats de travailleurs ruraux.

19 Quant à la nature du mouvement revendicatif, elle soulevait deux questions. Est-ce un mouvement social et populaire, ou est-ce un mouvement impulsé par des élites ? Si c'est un mouvement élitiste, pourrait-il devenir un mouvement social, avec une prise de conscience et une participation accrues de segments moins favorisés de la population - sans manipulation et sans cooptation de la part de la classe dominante, c'est-à-dire des leaders locaux?

20 Les nouveaux « municipes » - Eugenio de Castro, Entre-Ijuis et Sao Miguel das Missoes qui, avant de s'émanciper, constituaient trois des districts du «municipe » de Santo Angelo, ont réalisé un référendum pour leur émancipation à une même date, le 20 décembre 1987. Presque un an plus tard, ils ont réalisé des élections, le 15 novembre 1988, et les premiers maires et conseillers municipaux sont entrés en fonction le 1er janvier 1989.

Le Rio grande do Sul

21 Comme le processus d'émancipation n'est compréhensible qu'en fonction de la régionalisation de la zone, il est nécessaire d'apporter quelques éléments à ce propos. Traditionnellement reconnu comme un État qui repose sur les activités agro-pastorales, le Rio Grande do Sul a actuellement un revenu global qui n'est imputable que pour $17 \%$ à l'agriculture, et pour $55 \%$ aux services - d'après les études récentes de la Fondation de l'Économie et de la Statistique. Or on sait que la localisation du secteur tertiaire est essentiellement urbaine. Cette tendance à la tertiairisation est donc un facteur supplémentaire qui contribue à la concentration de la population dans les aires urbaines.

22 Le Rio Grande do Sul est grossièrement composé de deux zones historiquement et géographiquement distinctes : la campagne au sud, et le plateau au nord. La campagne, zone d'élevage extensif des ovins et des bovins, est traditionnellement une zone de faible densité démographique, avec un réseau urbain constitué de centres dispersés. Le plateau, région de polyculture, possède un réseau urbain plus dense, et c'est dans cette partie que se sont développées la plupart des villes de l'État. Ce fait est perceptible dans l'analyse des émancipations qui ont eu lieu jusqu'en $1966^{7}$ : pour la plupart, elles se situent dans la région du plateau, dans les zones de colonisation ancienne - celle de l'immigration allemande qui a commencé en 1825, et italienne qui a commencé plus tardivement, en 1874.

23 Au début des années quatre-vingt, le Rio Grande do Sul comptait 232 "municipes », nombre qui n'avait pas changé depuis 1966, par suite d'une loi promulguée sous le régime militaire. Ce nombre aurait probablement été plus élevé si, du fait de ces restrictions légales, le mouvement en faveur des émancipations n'avait été interrompu. C'est aussi par force de loi qu'il y a eu à partir de cette période une forte centralisation des ressources entre les mains du gouvernement fédéral, qui limitait beaucoup le pouvoir de décision des administrations locales quant à l'imputation de ce qui leur était 
rétrocédé. Il faut ajouter que toutes les communes de la frontière étaient soumises à la loi de la sécurité nationale, et qu'elles ne pouvaient même pas élire leurs maires : s'y substituait un intervenant directement nommé par le gouvernement central.

L'héritage centralisateur est ancien, et il ne se défait pas rapidement. Bien que nous vivions sous un régime de Fédération, les réclamations des administrations municipales et des administrations des États avaient trait non seulement à la fraction des ressources qui leur étaient rétrocédées, mais aussi à la possibilité de décider de leur imputation. Ceci explique en partie la difficulté que rencontraient les mairies, surtout dans les communes de superficie étendue, pour faire face aux besoins des districts, spécialement de ceux qui étaient les plus éloignés des chefs-lieux - et, partant, l'insatisfaction et le désir d'autonomie dans ces districts.

14 nouveaux « municipes » ont été creés en 1982, et en 1989 sont entrés en fonction les maires de 88 nouveaux "municipes" constitués à partir de districts qui se sont émancipés de leurs communes d'origine, après des consultations par voie de référendum durant l'année 1988. La plupart de ces nouvelles villes se situent sur le plateau. Cette région, qui est apparemment l'une des zones qui ont le plus perdu leur population par l'exode rural au profit d'autres États ou de la zone métropolitaine de Porto Alegre, continue malgré tout à être celle où les émancipations sont les plus nombreuses.

Un bref rappel historique

Un examen, aussi succinct soit-il, de la formation historique de la société du Rio Grande do Sul, montre que l'occupation la plus ancienne a été celle des Indiens guaranis, qui y vivaient librement jusqu'à l'arrivée des jésuites espagnols. Ceux-ci établirent sept " réductions ", les "Sete Povos das Missoes ", qui sont à l'origine des premiers noyaux de peuplement du XVII ${ }^{e}$ siècle, et qui ont été détruites par la suite lors de combats qui eurent pour origine des incursions des bandes armées originaires de Sao Paulo, qui venaient y chercher du bétail, du cuir et des esclaves.

Historiquement, la région a fait l'objet d'un litige entre Portugais et Espagnols, après la création par les jésuites espagnols de ces "Sete Povos das Missoes", où les Indiens guaranis, soumis à un processus civilisateur, s'adonnaient à l'agriculture et à l'élevage, mais aussi à l'art et à l'artisanat. Ils étaient ainsi très recherchés sur le marché des esclaves de la colonie, tout comme leur production qui fut à l'époque systématiquement pillée lors de luttes sanglantes, responsables de l'extermination quasi totale de la population indigène qui habitait dans cette région. San Miguel fut la capitale de ces " réductions » jésuites et on y trouve encore les ruines de l'ancienne cathédrale des missions.

Plus tard, au XIX ${ }^{e}$ siècle, les activités productives du Rio Grande do Sul rappellent encore la société pastorale d'origine dans la partie sud - la campagne, dont le peuplement a été longtemps compromis par des questions de frontières. L'autre partie - le plateau - voit l'établissement d'une société d'immigrants, de «colons». Celle-ci s'est toujours prioritairement adonnée à la polyculture, à l'artisanat et au commerce. Après l'extermination des Indiens et l'expulsion des jésuites, il faut ainsi attendre la fin du XIX ${ }^{e}$ siècle pour que le nord de l'État soit à nouveau occupé par un autre type de population, les familles des immigrants européens. Les lots, distribués dans le cadre de la politique d'immigration du gouvernement central, sont à l'origine des petites propriétés familiales consacrées à la polyculture qui prédominaient il y a encore peu dans tout le nord de l'État. C'est en partie cette structure foncière qui, plus tard, du fait 
des problèmes de division des propriétés lors des successions, poussa plusieurs générations à immigrer plus au nord, à mesure que s'épuisait la frontière agricole régionale ${ }^{8}$. Cette configuration historique initiale, et la situation excentrée du Rio Grande do Sul au sein du territoire national, expliquent sa base agro-pastorale traditionnelle, et son mode particulier d'intégration dans le marché national.

Si l'on suit l'évolution politico-administrative du territoire de l'État tout au long de son histoire, on peut observer un processus de fragmentation, depuis l'installation des jésuites en 1626, et l'installation du bourg de Rio Grande au sud-est en 1750, jusqu'à la création des quatre premières grandes zones en 1805 , qui rassemblaient alors tout le territoire de l'État. Chacune d'elles comptait un chef-lieu - Rio Grande, Rio Pardo, Santo Antonio da Patrulha et Porto Alegre.

31 Un saut dans le temps montre que le Rio Grande do Sul est passé en 178 ans de ces quatre grandes zones, qui couvraient toute l'immense étendue de l'État, aux 332 «municipes » actuels. La fragmentation continua de façon intermittente : elle fut plus rapide dans certaines périodes pour arriver, par suite de multiples divisions, aux communes actuelles; et elle fut toujours plus marquée dans les aires de colonisation vouées à la polyculture que dans les aires d'élevage tenues par des descendants des Portugais9.

32 La pulvérisation des communes s'est faite dans la "zone de la forêt ", où il y avait initialement une disponibilité d'espace vacant qui n'était pas occupé par des latifundia. C'est là qu'apparaît une classe moyenne, qui développe le secteur commercial, les communications et les transports, et qui se dissémine dans l'espace. Avec la mécanisation des cultures, une intégration entre les deux espaces - celui consacré à l'élevage, et celui dédié à la polyculture - commence à avoir lieu. La modernisation est ainsi liée au processus d'émancipation par deux facteurs essentiels : l'accroissement de la productivité, avec l'augmentation de la taille de la propriété, et l'exode rural ${ }^{10}$. L'un comme l'autre sont des effets de la mécanisation et sont étroitement liés à la structure foncière.

Dans les années soixante-dix, 110 communes de l'État ont perdu une part considérable de leur population, principalement dans la zone du plateau. Et l'on constate dès lors, simultanément, cette évasion de population d'un côté, et l'apparition d'une certaine prospérité pour ceux qui sont restés à la terre de l'autre. Certains d'entre eux - de petits agriculteurs - ont réussi à participer dès le début au processus de modernisation et, disposant initialement de quelques 25 hectares, ils ont pu augmenter leurs exploitations jusqu'à atteindre 150 ou 200 hectares, par l'achat des parcelles de ceux qui partaient ${ }^{11}$.

34 Ces facteurs expliquent pourquoi le nord du Rio Grande do Sul a été - et est encore aujourd'hui - une région de luttes pour la terre, où le « Mouvement des sans-terre » a toujours exprimé une tradition de résistance et une prise de conscience face aux injustices sociales.

35 Telle a été, d'une façon très sommaire, l'évolution de la région. Il faut maintenant nous demander ce que représente aujourd'hui, pour les descendants de ceux qui sont restés, restructurer un espace, organiser un nouveau "municipe ", former un nouveau cheflieu. Pour comprendre ces mouvements revendicatifs, on peut utilement les comparer à un iceberg, composé d'une partie cachée et d'une partie visible...

Les mouvements en faveur d'une émancipation : populaires ou élitistes? 

l'accent sur la nécessité d'implanter une infrastructure urbaine jusque là inexistante ou précaire (amélioration de la desserte routière à l'intérieur; meilleure proximité de l'offre de services - dispensaires et hôpitaux, écoles, agences bancaires, services fiscaux, etc.) Selon certaines des personnes rencontrées, c'est l'état d'abandon croissant des districts en faveur du chef-lieu qui fait que l'on aspire à l'indépendance administrative. Il y a aussi l'attente, quelquefois explicite, que l'émancipation provoque la création d'emplois urbains susceptibles de retenir ou de faire revenir les enfants partis étudier ailleurs, et qui finissent par ne pas s'établir dans la localité. De toute façon, le fait frappant est la quasi-inexistence d'opposants directs au mouvement en faveur de l'émancipation - sauf dans les zones situées à proximité des limites préconisées, quand des districts y nourrissent eux-mêmes le désir de s'émanciper un jour, et craignent que leur adhésion ne constitue alors une entrave.

Pour des raisons politiques - partisanes ou non -, les intérêts de ceux qu'on appelle les leaders locaux divergent. Parce qu'il détient tous les services administratifs de la commune, le chef-lieu a droit à davantage de dépenses publiques. Comme c'est aussi pour des raisons politiques que la distribution des ressources est inégale - que ce soit entre les divers districts d'un même " municipe », ou entre ces districts et le chef-lieu -, un argument fort, pendant la campagne en faveur de l'émancipation, consiste à insister sur l'état d'abandon du district et à démontrer que "le chef-lieu exploite le district ", en présentant les ressources dont le chef-lieu dispose comme autant d'impôts levés. Selon l'un de nos interlocuteurs, comme il n'y a pas de rétrocession sur ce qui a été collecté, les districts sont perçus comme assurant la maintenance de la "métropole ».

répand ainsi une sorte d' "idéologie du territoire ». Mais selon un témoignage, «ce que les leaders font en réalité, c'est qu'ils canalisent le mécontentement de la population. Car ces gens de l'intérieur n'ont jamais eu la pratique de l'engagement dans des luttes. L'intérieur est beaucoup plus conservateur que la ville. Ils sont fidèles aux partis : "Moi je suis du parti X. »

discours relatif au bien public, apparait la contradiction de ce processus en faveur de l'émancipation, qui exprimerait apparemment un désir populaire actif. Certains témoignages font clairement état d'un processus élitiste : "Ce sont les élites qui commandent et le peuple n'est consulté qu'après. Il n'y a pas de discussion avec la population." Ou encore: "Ici, le pouvoir a toujours été aux mains de ceux qui sont avec le gouvernement. Il s'est donc créé un très fort clientélisme politique dans la région. Et puis l'absence de contrôle sur le budget communal facilite la manipulation des ressources afin de financer des campagnes électorales. "

Dans le discours, l'intérêt des élites est au service du "bien public », objectivé dans l'obtention d'infrastructure, ce qui explique la quasi-inexistence de voix discordantes. Les intérêts politiques (l'attribution des futurs postes dans le gouvernement local), et économiques (le lotissement urbain par exemple), restent implicites, sous-jacents, masqués, recouverts. Autrement dit, les granjeiros ${ }^{12}$ sont favorables à l'émancipation parce qu'il y aura une amélioration des infrastructures urbaines, et que les terres, rurales et urbaines, seront valorisées par les infrastructures publiques qui suivront la création du «municipe ».

41 Il y a aussi des intérêts indirects, liés à la socialisation de certaines dépenses. Ceux qui sont propriétaires de terrains ou de lotissements urbains - ou qui désirent le devenir veulent acquérir leurs propriétés ou régulariser leur obtention grâce à l'extension du périmètre urbain et à l'approbation d'un plan directeur urbain qui permette tout à la 
fois de valoriser le prix du foncier, et de reporter sur l'État, par l'intermédiaire de la mairie, les coûts des infrastructures (électricité, eau, percée de rues, etc.) Pour plusieurs des personnes rencontrées, il est clair que les commerçants et ceux qui ont beaucoup de terres profitent de l'émancipation - les uns par l'augmentation du flux des transactions commerciales, les autres par la valorisation de leurs terrains.

S'y ajoute, pour les granjeiros, que les dépenses relatives à la santé et à l'assistance directe aux employés incomberont désormais à l'État. Ce transfert à l'État devient effectif quand, pour la création d'une nouvelle ville, on peut réclamer avec plus de force l'installation de postes de santé ou de dispensaires médicaux financés sur des ressources publiques.

Par connivence ou par naïveté, certains nient l'existence de ces intérêts dans le processus en faveur d'une émancipation, affirmant qu'il est spontané et sincère. D'autres déclarent qu'il existe des intérêts particuliers, mais ils n'en ont pas une perception claire. La quasi-inexistence d'opposants, bien que plusieurs personnes soient conscientes des distorsions du processus politique de formation du consensus, s'explique en partie par les carences objectives réelles, et par les désillusions déjà subies au cours du processus de participation populaire.

Tout ceci apparaît encore plus nettement quand, à l'examen des intérêts en jeu, on ajoute celui des avantages et désavantages des émancipations, tels qu'ils sont perçus par la population interviewée avant les référendums. Les désavantages mentionnés se rattachent fondamentalement à la crainte d'une augmentation des impôts. Par ailleurs, les petites localités situées à la périphérie, et donc distantes du futur chef-lieu, ne voient pas d'avantage à s'intégrer dans une nouvelle commune qui, outre sa distance, ne disposera encore pendant un temps que d'une infrastructure précaire. Leur intégration pourrait aussi constituer un obstacle à leur future émancipation. Cependant, ceux qui évoquent ces réserves ne sont pas opposés à l'idée que les districts qui le demandent deviennent de nouveaux « municipes » : "Ca va être bien pour nous. Ca va donner de meilleures ressources. Toute la région va se développer... " Mais ils craignent aussi de ne plus pouvoir bénéficier des machines assurant l'entretien des routes, qui appartiennent à la commune d'origine. Leur desserte deviendrait ainsi encore plus difficile.

L'argument le plus rationnel en faveur des émancipations, c'est qu'administrer une commune de petite taille est préférable et plus aisé. Pour les agriculteurs de l'intérieur, l'amélioration des routes, favorisant l'évacuation de la production et l'accès à la ville, est un argument convaincant, ainsi que l'implantation éventuelle d'écoles pour leurs fils. Une autre aspiration des ruraux est l'installation dans la nouvelle ville d'une banque pour le financement des cultures, et pas seulement pour la gestion de comptes courants.

Quant aux habitants des chefs-lieux, leurs attentes sont multiples. Les petits commerçants espèrent que leurs affaires vont se développer. Pour d'autres, des jeunes principalement, être un "municipe" est un élément qui jouera en faveur de l'installation d'industries et de commerces, avec des emplois à la clé. Ils nourrissent l'espoir que, plus tard, l'implantation d'une faculté permettrait de poursuivre des études. On argue aussi que la résolution des problèmes sera facilitée avec des maires et des conseillers municipaux plus proches.

Ce qui est le plus souvent mis en avant par la population rurale est aisément compréhensible pour qui connaît la réalité hors des grandes villes du Brésil : c'est 
l'installation d'une infrastructure de santé - postes de santé, dispensaires, services dentaires, pharmaceutiques. Vient ensuite l'attente relative à l'installation d'écoles pour les enfants et les adolescents.

Quelques éléments de réflexion

La «régionalisation" étant comprise comme le processus qui transforme continuellement la réalité régionale, de multiples réflexions sont possibles, car la question étudiée se situe à l'interface de très nombreuses autres questions. Deux grands ordres de réflexion vont être privilégiés ici : les transformations de la structure régionale productive, la régulation des relations sociales.

Les transformations de la structure productive régionale

La modernisation et l'implantation de la monoculture ont contribué à transformer la structure foncière régionale. D'une région de petites propriétés familiales vouées à la polyculture, celle-ci se transforme petit à petit en une région constituée pour une part de minifundia, pour une autre d'un nombre croissant de moyennes et grandes propriétés. Dans cette région, qui depuis des décennies est une zone d'émigration, ceux qui restent deviennent des minifundistes - dont les terrains accidentés gênent la mécanisation - ou des sans-terre, ou bien ils s'affirment comme des entrepreneurs ruraux de moyenne dimension, liés à des activités urbaines.

50 Les habitudes de travail du producteur ont aussi été modifiées. Auparavant, au sein de chaque unité productive, des moyens d'autosubsistance étaient produits: fruits et légumes, élevage, produits laitiers, etc. Toutes ces activités demandaient un temps de travail important. Elles se sont transformées avec la monoculture qui n'exigea plus que la moitié du temps occupé antérieurement - d'autant que la mécanisation a entraîné la quasi-extinction du travail manuel.

51 Tout ceci incite à penser que dans le Rio Grande do Sul, et surtout dans la région étudiée, contrairement à ce qui se passe dans d'autres régions d'exploitation agricole plus récente, le revenu produit est inférieur aux nécessités de l'infrastructure. A partir de là, on pourrait se demander si le placement des excédents ne s'effectuerait pas hors de la région, dans des secteurs plus lucratifs, au détriment des besoins régionaux processus dont les effets sont déjà visibles dans l'implantation de taudis à la périphérie des villes moyennes ou au long des routes, ou dans les campements des gens sans terre, qui sont fatigués des promesses de l'État, et menacés par la faim, le froid et les maladies.

La régulation des relations sociales

52 Au Brésil, divers travaux sur le pouvoir local ont montré combien les moins favorisés sont soumis au clientélisme - dont l'œuvre de Leal, qui fait figure de classique ${ }^{13}$. D'autres démontrent que les dites «élites ", la classe dominante, cherchent à socialiser leurs coûts privés en profitant de l'État qu'ils transforment en État "patrimonialiste » ${ }^{14}$. D'autres enfin démontrent la continuité de ces élites, sans transformation profonde de leurs intentions ${ }^{15}$.

53 Suivre le mouvement pour la création de nouveaux chefs-lieux de "municipes » a permis de percevoir la persistance du vieux style clientèliste, et de repérer comment il s'insère dans un usage politique effectif du territoire. Les fractions dominantes de la population régionale engagées dans la modernisation souhaitent vivement la création de "l'État local » pour se transformer en une fraction gouvernante locale. C'est à cette fin que ces élites - ou leurs préposés - sont à l'initiative des émancipations et à la tête 
des commissions qui en sont chargées, au nom du bien public, contre lequel on ne peut s'insurger.

Pour cela, ils se prévalent d'un discours sur l'espace qui vise tout à la fois la formation d'hégémonies et d'alliances dans les nouveaux chefs-lieux, la tutelle du peuple au nom du progrès et du développement, et la diffusion d'une idéologie territoriale. La création de «l'État local » permet de placer dans les secteurs urbains les plus valorisés (terrains, lotissements) les excédents engendrés par la modernisation, en même temps qu'elle procure une infrastructure urbaine capable de renforcer le processus de modernisation (routes, écoles, santé, banques, organismes publics). Elle signifie aussi un transfert des dépenses sociales à l'État.

C'est ainsi que s'établissent les relations de pouvoir, l'espace étant un instrument, et la ville le lieu où se forgent les nouvelles hégémonies ${ }^{16}$. Il s'agit de fait d'un véritable usage politique du territoire.

Citoyenneté et émancipations

Un autre aspect de cet usage politique du territoire concerne la relation entre l'exercice de la citoyenneté et les émancipations. Au Brésil, d'une façon générale, on est peu habitué à prendre part aux décisions - en grande partie par suite des motifs déjà indiqués. On dépose son bulletin de vote dans l'urne, et on délègue ainsi totalement aux élus toute la responsabilité et tout le pouvoir de décision relatif à la ville, à l'État, au pays, sans mécanismes de contrôle a posteriori. L'apathie manifestée par beaucoup lors des processus d'émancipation ne déroge pas à la règle, ni à la tradition élitiste de gouverner. Comme l'affirme Correa da Silva, "La lutte des masses au Brésil, c'est une lutte pour la formation de citoyens. Le problème consiste à créer une société civile forte ${ }^{17}$ ».

D'autre part, pour contradictoire que cela puisse paraître, on observe non seulement un mouvement croissant en faveur de la municipalisation ${ }^{18}$, pour plus d'autonomie et par conséquent pour un plus grand renforcement de l'unité administrative, mais aussi un essor du mouvement associatif, qui existait auparavant et s'était trouvé récemment en récession. Les associations administratives créées dans l'année qui a suivi la mise en place des nouveaux chefs-lieux - ou même auparavant - sont innombrables. L'« associativisme » formel est une tradition très forte qui semble être stimulée par la FAMURS ${ }^{19}$. Outre les associations régionales regroupant les maires, existent des associations de secrétaires de gouvernement municipal, de conseillers municipaux, de forums régionaux, etc. Il existe aussi, semble-t-il, un associativisme informel d'aide entre des communes proches.

Ces éléments expliquent pourquoi les stratégies territoriales élaborées pour la création de nouvelles communes ne vont pas au-delà des limites étroites et ambitieuses d'intérêts politiques et économiques, ou d'objectifs collectifs à très court terme relatifs à l'implantation d'infrastructures. Autant qu'on en puisse juger, il n'y a pas de vision à long terme. L'intérêt pour un débat relatif à un projet de société est quasi inexistant. Des thèmes comme la question agraire, la modernisation et ses effets, la diversification de l'agriculture, restent du domaine du clergé, des syndicats de travailleurs ruraux, des forums alternatifs des mouvements écologiques, ou des analyses des milieux scientifiques.

59 Les émancipations, avec la formation de nouvelles communes, semblent représenter de nos jours ce qu'était la politique des districts industriels dans les années soixante-dix: 
un moyen pour les «municipes» d'obtenir une rétrocession de ressources, qui sont toujours rongées par l'inflation.

L'une des questions qui se posent aujourd'hui à la société brésilienne est sans doute de penser cette nouvelle urbanisation qui ne se réalise pas seulement en tache d'huile, à partir des grandes métropoles, comme l'entendait Lasuen dans les années soixante-dix. Cette urbanisation cache encore les anciennes façons de faire clientèlistes et autoritaires sous les habits d'une démocratie discriminatoire et d'une apparente plus grande participation.

Dans le jeu des futurs possibles, il faut donc chercher des modalités d'exercice de la citoyenneté qui, avec la création des nouvelles villes, rendent possible l'émergence de nouvelles façons de faire de la politique: permettant plus d'agrégation, plus de solidarité, elles reposeraient sur une éthique différente de celle qui, jusqu'ici, est à la fois l'origine et la conséquence des injustices sociales.

\section{NOTES}

1. Circonscriptions administratives autonomes, les municipios sont gouvernés par un maire (prefeito) et des membres d'une chambre municipale. Les cidades sont les chefslieux des municipios (NdE).

2. Cf. Zila MESQUITA, 1988, « Os « espaços » do espaço brasileiro em fins do seculo XX », Terra livre, 4, Sao Paulo, Associaçao dos geografos brasileiros AGB, pp. 9-38.

3. Cf. Alberto Passos GUIMARAES, 1978, A crise agraria, Rio de Janeiro, Paz e terra ; Argemiro BRUM, 1985, Modernizaçao da agricultura : trigo e soja, Ijui, FIDENE ; Geraldo MULLER, 1985, " A velha senhora agraria e seus novos balangandas », Novos estudos CEBRAP, 11, Sao Paulo, pp. 2-9 ; Ariovaldo Umbelino OLIVEIRA, 1987, Amazonia : monopolio, expropriaçao e conflitos, Campinas, Ed. Papirus.

4. Cf. Zila MESQUITA, art. cit. , p. 11.

5. Cf. Argemiro BRUM, op. cit.

6. Cf. Zila MESQUITA, 1984, « Divisoes regionais do RS : uma revisao », Ensaios FEE, Porto Alegre, Fundaçao de economia e estatistica, 5 (2), p. 97.

7. Cf. Gildo WILLADINO, 1959, « Municipalizaçao no Rio Grande do Sul », Boletim geografico do Rio Grande do Sul, Porto Alegre, CEMAPA, 4 (9/10); Flavia LA SALVIA et Elisabeth MARODIN, 1976, "Evoluçao municipal : uma analise geografica », Boletim geografico do Rio Grande do Sul, Porto Alegre, CEMAPA, 21 (19), 3-16 jan/déc.

8. Jean ROCHE décrit magistralement ce processus dans son étude de la colonisation allemande. Cf. A colonizaçao alema e o Rio Grande do Sul, Porto Alegre, Ed. Globo, 1969.

9. Cf. Zila MESQUITA, art. cit. , 1984, pp. 98-99.

10. Entretien avec l'ingénieur-agronome A. TERHOSTde la Coopérative chargée de la commercialisation du blé de Santo Angelo -COTRISA-, en janvier 1988.

11. Entretien avec Argemiro BRUM, Professeur à la UNIJUI, 7/1/1987.

12. Granjeiro est le terme régional désignant les détenteurs de propriétés rurales de moyenne et grande dimension. 
13. Cf. Victor Nunes LEAL, 1949, Coronelismo, enxada e voto. $O$ municipio e o regime representativo no Brasil, Sao Paulo, Ed. Alfa-Omega, 3è édition 1976.

14. Cf. Simon SCHWARTZMAN, 1988, Bases do autoritarismo brasileiro, Rio de Janeiro, Ed. Campus.

15. Cf. BURSZTYN, 1990, O pais das alianças, Elites e continuismo no Brasil, Rio de Janeiro,

Ed. Vozes Petropolis.

16. Bertha BECKER, Professeur à la UFRJ, dans une communication à l'Instituto Panamericano de Geografia e Historia, IPGH, Rio de Janeiro.

17. Entretien avec Armando CORREIA DA SILVA, Professeur au département de géographie, USP, janvier 1987.

18. Ce qui avait déjà été noté par Beatriz MANICA, Professeur à la FUNDAMES, se référant à la création de conseils municipaux au sein des communes existantes, en janvier 1988.

19. Fédération des associations de « municipes » du Rio Grande do Sul, à laquelle est affiliée l'Association des « municipes » de la Région Missioneira, qui comprend les 18 «municipes » de cette région.

\section{RÉSUMÉS}

La dernière décennie a vu l'émergence de divers mouvements luttant en faveur d'une «émancipation » locale par la création de nouvelles communes, et contribuant à une nouvelle urbanisation qui ne se réalise pas à partir des grandes métropoles. S'agit-il de mouvements populaires, ou de processus impulsés par les élites locales? L'analyse de trois cas d'émancipation dans le Rio Grande do Sul permet de percevoir la persistance de l'ancien clientélisme, et de repérer comment il s'insère dans un usage politique du territoire.

The new "municipios" in Brazil: three cases of emancipation process in an area of agricultural modernization.

During the last decade, various movements have been looking for local emancipation, contributing besides to a new kind of urban growth apart from metropoles. Three processes of emancipation in the Rio Grande do Sul region are analysed. They allow to perceive the persistence of old « clientélisme » and to discover how this old system is adapted to fit into a new political use of territory.

\section{INDEX}

Mots-clés : Développement local, pouvoir local, Urbanisation, Brésil

Keywords : Local development, local power system, Brazil 
AUTEUR

ZILA MESQUITA

Professeur au Département de géographie de l'Université Fédérale de Rio Grande do Sul (Brésil). 